\title{
The Treatment of Sudeck's Syndrome in the Upper Extremities with Sympathetic Blockage by Using Ultrasound
}

\author{
Mustafa Guler, M.D., ${ }^{\star}$ Cetin Onder, M.D., ${ }^{\star *}$ Celal Baki, M.D., ${ }^{\star *}$ Mehmet Kirnap, M.D.* \\ Gurcan Uremek, P.T., ${ }^{\star}$ Halit R. Gumele, M.D. ${ }^{\star * *}$ \\ Trabzon, Turkey
}

DOI: http://dx.doi.org/10.5915/23-4-15414

\begin{abstract}
Sudeck's syndrome is a disease of pain, swelling, hyperesthesia, hyperhydrosis and trophic changes in the skin and bone of the affected distal extremity. A number of diseases, precipitating events, and drugs have been associated with Sudeck's syndrome. It is usually believed that the etiological factors produce pathologic changes in the neuro-vascular system. The vicious cycle of pain-spasm-anoxia causes the problem to become chronic. In therapy, it is believed that if the pain disappears, the pathophysiological vicious cycle has been stopped. For this reason, a number of medical therapy modalities and physical therapies can be used. In this study we used the ultrasound as a sympathetic ganglion blocker for the therapy for patients with Sudeck's syndrome.
\end{abstract}

Key words: Sudeck's syndrome, ultrasound, sympathetic blockage.

Sudeck's syndrome (Reflex Sympathetic Dystrophy or RSD), is a clinical entity characterized by trophic and vasomotor changes, limitation of motion, and pain which usually appears after trauma. ${ }^{1,2}$ This syndrome was first described by Mitchell in 1891 , and termed "causalgie." Then, Sudeck in 1900 described the syndrome in detail. ${ }^{3}$

RSD can be due to trauma of the soft tissues, fractures, dislocations, prolonged immobilization, surgery, disturbances of circulation, myocardial infarctions and head traumas. In all cases, it is believed that the final etiologic factor is a pathologic change

From the Department of Physical Medicine and Rehabilitation*

Orthopedic and Traumatology, ** and Radiology, *** Karadeniz Technical University, Trabzon, Turkey

Reprint Requests: Mustafa Guler, M.D.

Department of Therapy and Rehabilitation

K.T.U. Tip Fakultesi, Farabi Hastanesia, F.T.R.

Anabilim Dali, 61080 Trabzon, Turkey in the neurovascular system, that will cause trophic changes in the muscles of the extremity. The vicious cycle of pain-spasm-anoxia causes the cases to become chronic. ${ }^{4,5}$

Different methods have been used for the treatment of the disease. In therapy, it is believed that if the pain disappears, then the pathophysiologic cycle has stopped. Anti-inflammatory agents, analgesic drugs, and physical therapy equipments (low and medium frequency curves, ultrasound) can be used. To regulate the disturbances of the circulation, the blockage of the stellate ganglion by medical therapy, electric currents or ultrasound can also be tried. ${ }^{6,}$ ?

\section{Materials and methods}

This study was performed at the Kastamonu Rehabilitation Center, the Physical Medicine and Rehabilitation, and the Orthopedic and Traumatology Departments of Karadeniz Technical University School of Medicine. Twenty-four patients with Sudeck's syndrome were studied. The patients were divided into two groups. The first group received hot-packs, exercises and ultrasound on the stellate 
Table 1. Clinical data of the study patients:

A. First group

\begin{tabular}{cccl}
\hline Number & $\begin{array}{c}\text { Age } \\
\text { (Yrs) }\end{array}$ & Sex & \multicolumn{1}{c}{ Etiology } \\
\hline 1 & 23 & M & Trauma (Right elbow) and immobilization \\
2 & 28 & M & Dislocation (Right elbow) \\
3 & 56 & M & Arthrosis (Left shoulder) \\
4 & 13 & F & Trauma (Right elbow) and immobilization \\
5 & 50 & F & Idiopathic (Right wrist) \\
6 & 34 & M & Fracture (Right elbow) \\
7 & 19 & F & Trauma (Left elbow) and immobilization \\
8 & 38 & M & Trauma (Right elbow) \\
9 & 37 & M & Trauma (Left elbow) and immobilization \\
10 & 31 & M & Cervico-brachial neuralgia (Right) \\
11 & 33 & F & Trauma (Right wrist) \\
12 & 65 & F & Dislocation (Right elbow) \\
\hline
\end{tabular}

B. Second group

\begin{tabular}{rccl}
\hline Number & $\begin{array}{c}\text { Age } \\
\text { (Yrs) }\end{array}$ & Sex & \multicolumn{1}{c}{ Etiology } \\
\hline 1 & 60 & M & Trauma (Left hand) and immobilization \\
2 & 65 & M & Fracture (Left elbow) \\
3 & 16 & M & Fracture (Left elbow) \\
4 & 56 & M & Trauma (Right wrist) and immobilization \\
5 & 40 & F & Trauma (Left wrist) and immobilization \\
6 & 50 & F & Periarthritis (Left shoulder) \\
7 & 65 & F & Periarthritis (Right shoulder) \\
8 & 55 & F & Frozen shoulder (Right) \\
9 & 43 & M & Fracture (Left clavicula) \\
10 & 52 & M & Arthrosis (Left shoulder) \\
11 & 23 & F & Idiopathic (Left shoulder) \\
12 & 30 & M & Dislocation (Left shoulder) \\
\hline
\end{tabular}

ganglion as therapy. The second group received only hot-packs and exercises.

The patients were chosen among those without cerebrovascular disease, hypertension, infection, hypoesthesia or any tumoral structure at the region of the ultrasound application. A point $3 \mathrm{~cm}$ above the sternoclavicular joint was chosen as the localization site of the stellate ganglion. Ultrasound was applied to the stellate ganglion once a day, for 10 days. The dosage was $1 \mathrm{watt} / \mathrm{cm}^{2}$ for five minutes at each session. Hot-pack applications lasted 20 minutes for each patient, and then passive and active exercise programs were introduced for 20 minutes.

Clinical findings such as pain, sensibility, hyperesthesia, edema, limitations in the motion of shoulder and the finger joints were evaluated before and after the therapy. Results were rated with respect to their intensity as: absent (0), weak (1), medium (2), severe (3), and very severe (4). The mean score for each of these symptoms in the two groups were calculated both before and after therapy. The means were compared using student " $\mathrm{t}$ " test

\section{Results}

The clinical data of the study patients are given in table 1.

Ten $(41.7 \%)$ of the patients were female and 14 $(58.3 \%)$ were male. The etiologic factors were; trauma: nine $(37.5 \%)$, dislocation of the shoulder joint: three $(12.5 \%)$, idiopathic: two $(8.3 \%)$, arthrosis and periarthritis of the shoulder: five $(20.8 \%)$, cervicobrachial neuralgia: one $(4.2 \%)$, and prolonged immobilization of joints due to fractures: four $(16.6 \%)$, respectively.

In the first group, pain improved at the rate of $80 \%$ (the score was 3.16 before therapy, and 0.66 after therapy). When it was compared with the recovery rate of $32 \%$ in the second group, the result was found to be statistically significant $(P<0.05)$.

The recovery rate of sensibility was $62 \%$ in the first group (the score was 3.25 before therapy, and 1.25 after therapy) and it was $19 \%$ in the second group (the score was 2.66 before therapy, and 2.16 after therapy). The difference was statistically significant $(\mathrm{P}<0.05)$. 
The recovery rate of edema in the first group was $76 \%$ (the score was 3.16 before therapy, and 0.75 after therapy) and in the second group it was $28 \%$ (the score was 3.0 before therapy, and 2.16 after therapy). The difference was statistically significant $(\mathrm{P}<0.05)$.

Limitation of joint motions showed recovery at the rate of $73 \%$ (the score was 2.75 before therapy, and 1.83 after therapy) in the first group and $31 \%$ (the score was 2.66 before therapy, 1.83 after therapy) in the second group. The difference between the two groups was statistically significant. $(\mathrm{P}<0.05)$.

\section{Discussion}

Although some factors are thought to be causative, the etiology of Sudeck's syndrome (RSD) is still unknown. In our cases, we established trauma, dislocation and arthrosis of the shoulder, cervical arthrosis (cervico-brachial neuralgia), prolonged immobilization and idiopathic causes as etiological factors. Similar factors have been mentioned in the literature. ${ }^{8}$ In our study, fractures, dislocations and trauma were the most common. However, soft tissue trauma was the most common factor for Sudeck's syndrome in another study. 9

Blonchi et $\mathrm{al}^{10}$ determined that the syndrome affects female patients, and affects the right side more frequently. In our study $41.7 \%$ of the patients were females. We did not find any difference between the right and the left side involvement. The risk of RSD is reported to increase with age. The mean age was 41 years, while it was 40 , in another study. ${ }^{11}$

The syndrome usually affects the upper extremities. The knee or foot is rarely affected. ${ }^{12}$ In our patients only the upper extremity was involved.

Although there are various concepts for the pathogenesis of RSD, the sympathetic hyperactivity is believed to be the most important factor. ${ }^{13}$ Some investigators have mentioned the importance of this as a spinal cord response to painful stimuli. Others asserted that the sympathetic hyperactivity arises at the higher sympathetic centers of the brain. ${ }^{14}$ De Takats, ${ }^{15}$ Steinbrocker ${ }^{16}$ suggested that soft tissue injuries cause degeneration of the neurons, and Moberg mentioned the importance of venous or lymphatic changes as a basis of the beginning of disturbances. ${ }^{17}$ The usual clinical findings of sympathetic hyperactivity such as hyperesthesia, pain, hypersensibility, hyperhydrosis were recorded in our cases.

Typically, the laboratory analysis does not show any important changes. There was X-ray evidence of mild or moderate osteoporosis in $75 \%$ of our cases.

In the treatment of RSD, it is believed that the sooner the therapy, the higher will be the rate of success. Rosen and Graham reported $43 \%$ recovery in cases of less than six months duration, and $20 \%$ in cases of more than six months duration. ${ }^{18}$ In 14 $(58.3 \%)$ of our cases the duration of the disease rang- ed from three to six months, in seven $(29.2 \%)$ the duration of the disease was less than three months, and in three $(12.5 \%)$ the duration of the disease was longer than six months.

Anti-inflammatory drugs, hot and cold application, exercise, radiotherapy, Bier's blockage, acupuncture, transcutaneous electric nerve stimulation (TENS), thalamotomy and hypnosis have been used as therapeutic approaches for the Sudeck's syndrome. The effectiveness of these therapies has been found to vary..$^{6,7,11,19}$

Anti-inflammatory drugs are effective in decreasing the pain, edema and inflammation. Because of their high anti-inflammatory properties, the steroids (prednisone, $60-80 \mathrm{mg} /$ day for 2-3 weeks) have been given to the patients with RSD and with recovery rates of $60-75 \% .^{7}$

Sympathetic ganglion blockage with a number of therapeutic modalities results in different rates of recovery ranging from 25 to $100 \%$. Patman et al have obtained good results by using sympathetic blockage in 41 patients with RSD, but most of them needed sympathectomy after a while. ${ }^{20}$ Homans has reported $80 \%$ recovery by the blockage of paravertebral ganglia. ${ }^{21}$ Also, some investigators have asserted that paravertebral ganglionectomy and periarterial sympathectomy were the most effective and definitive methods in the therapy of Sudeck's syndrome. ${ }^{21,22}$

Ultrasound is usually used for its deep heat and micromassage effects. More recent studies have demonstrated that low doses of ultrasound cause excitation of the nervous system, medium doses cause inhibition and analgesia and high doses cause destruction. $^{22,23}$ Several investigations $\mathrm{s}^{24-27}$ have observed a decrease of arterial blood pressure by applying ultrasound to the stellate ganglion. They observed an increase in the temperature of the skin after application of ultrasound. Yanlioglu has determined that ultrasound causes increase in the temperature of the skin and of the blood pressure by affecting the sympathetic nervous system. ${ }^{28}$

In our study, we tried to treat the disease by applying ultrasound in order to block the ganglion without using anti-inflammatory or analgesic drugs. We found it to be effective. We observed higher rates of recovery in the group which received ultrasound therapy compared with the group which did not. These results show that ultrasound can be used as a sympathetic blocker, and that it is effective in the therapy of RSD.

\section{References}

1. Kozin, F: Painful shoulder and RSD Syndrome. In: Mc Carty DJ, ed. Arthritis and Allied Conditions. Ninth edition, Philadelphia: Lea Febiger 1979; 1111-7.

2. Weis, J: The Painful shoulder. In: Kelly W. Har- 
ris E.D. Ruddy S, Sledge C.B: Textbook of Rheumatology. Philadelphia: WB Saunders 1981; 437-55.

3. Sudek P: Die trophische extremitantens groung durch periphere (Infetoise and traumatische), Reize, Dtsch 2 Chir 1931; 234: 596-612.

4. Schwartzman, RJ, Lellen, TL: Reflex Sympathetic Dystropy Arch Neur 1987; 44: 555-61.

5. Poplawski, J, Wiley, AM, Murray, UP: Posttraumatic dystrophy of the extremities. Am J Bone Surg 1983; 65: 642-55.

6. Schutzer, SF, Gossling, HR: The treatment of RSD Syndrome. J Bone Joint Surg 1984; 66: 625-29.

7. Glick, EN: Reflex Dystrophy. Results of treatment by corticosteroids. Rheumatol Rehabi 1973, 12: 84-8.

8. Kozin, F, Mc Carty, DJ, Sims, J, Genant, H: The Reflex Sympathetic Dystrophy Syndrome, Am J Med 1976, 60: 321-30.

9. Pak, TJ, Martin, GM, Magner, JL: Reflex sympathetic dystrophy, Review of 140 cases. Minn Med 1970; 53, 507-12.

10. Blonchi, C: La terapia fisica nella sindrome dr Sudeck. Francastora 1968; 61: 748-63.

11. Hannington-Kif, JG: Relief of Sudeck's atrophy by regional intravenous guanethidine. Lancet 1977; 1: 1132-3.

12. Tietjen, R: RSD of the knee, Clin Ortho Rel Res 1986; 209: 234-43.

13. Devor, M: Nerve pathophysiology and mechanisms of pain in Causalgia. J Auton Nerv Syst 1983; 7: 371-84.

14. Noordenhous, W: Pain. Amsterdam, Elsevier Press, 1949; 21-36.

15. De Takats, G: The nature of painful Vasodilatation in Causalgic states. Arch Neurol 1943; 50 . 318-26.

16. Steinbrocker, O: The shoulder-hand syndrome, Present perspective. Arch Phys Med Reh 1968;
49: 388-94.

17. Moberg, E: Surg Clinic N Amer 1960; 40: 369-72.

18. Rosen, PS, Graham, W: The shoulder hand syndrome with observations on 73 patients. Historical review. Canad Med Assoc J 1957; 77: 86-91.

19. Cinar, A, Aktas, S: Therapy with diadinamic currents in patients with Sudeck's syndrome. Ataturk Univ Bull 1979; 11(3): 392-9.

20. Patman, RD, Thompson, JE, Persson, AU: Management of posttraumatic pain syndrome, Report of 113 cases. Ann Surg 1973; 177: 780-7.

21. Homans, J: Minor Causalgia, a hyperesthetic neurovascular syndrome. N Engl J Med 1940; 222: $870-4$.

22. Kocas, H: The effect of ultrasound on the neurovegetative system. The thesis of Associate Professor, Ankara, 1971, 3-4.

23. Harold, FS. Consideration in ultrasound therapy and equipment performance. Physical Therapy 1980; 69: 11-7.

24. Bidwai, AV, Rogers, CR, Pearce, M, Stanley. TH: Preoperatives stellate ganglion blockade to prevent hypertension following coronary artery operations. Anesthesiology 1979; 51: 345-7.

25. Fee, HJ, Viljoen, Cukingnan, RA, Canas, MS: Right stellate ganglion block for treatment of hypertension after cardiopulmonary bypass. Annals Thor Surg 1979; 27: 519-21.

26. Estefanous, GF: Preoperative stellate ganglion blockade to prevent hypertension following coronary-artery operations. Anesthesiology, 1980; 52: 454-5.

27. Cureklibatir, F: The effect of ultrasound on the pulse and blood pressure when applied to ganglion stellare. Ege Univ Med 1980; 19:437-43.

28. Yanlioglu, N: The effects of Ultrasound on autonomic nervous system, The thesis of associate professor, Ankara 1966; 47-55. 